\title{
Increased expression of tight junction protein occludin is associated with the protective effect of mosapride against aspirin-induced gastric injury
}

\author{
CHENCHEN LIU $^{1,2^{*}}$, ZHAOTAO DUAN $^{1 *}$, YUE GUAN $^{1}$, HAILU WU $^{1}$, KEWEI HU $^{1}$, XIN GAO $^{1}$, \\ FANGCEN YUAN $^{1}$, ZONGDAN JIANG $^{1}$, YE FAN ${ }^{1}$, BANGSHUN HE ${ }^{3}$, SHUKUI WANG $^{3}$ and ZHENYU ZHANG ${ }^{1}$ \\ ${ }^{1}$ Department of Gastroenterology Nanjing First Hospital, Nanjing Medical University, Nanjing, Jiangsu 210006; \\ ${ }^{2}$ Department of Gastroenterology, Jining First People's Hospital, Jining, Shandong 272111; ${ }^{3}$ General Clinical \\ Research Center, Nanjing First Hospital, Nanjing Medical University, Nanjing, Jiangsu 210006, P.R. China
}

Received July 26, 2015; Accepted January 20, 2017

DOI: 10.3892/etm.2017.5550

\begin{abstract}
Mosapride is known to affect gastric motility, however whether mosapride has anti-ulcergenic effects in gastric mucosal injury is unclear. The aim of the present study was to investigate the effects of mosapride on aspirin-induced gastric injuries. GES-1 cells were cultured and divided into 5 groups: Control group, aspirin injury group (treated with $18.2 \mathrm{mmol} / 1$ aspirin) and mosapride pretreatment groups (treated with $0.4,0.5$, or $0.6 \mu \mathrm{mol} / 1 \mathrm{mosapride)}$. Cell proliferation was evaluated via MTT assay and cell apoptosis was investigated via flow cytometry. The expression of occludin was determined by western blot analysis. A total of 40 male Sprague-Dawley rats were randomized into five groups: Control group, aspirin injury group $(150 \mathrm{mg} / \mathrm{kg})$ and mosapride pretreatment groups $(0.25,0.50$ or $0.75 \mathrm{mg} / \mathrm{kg})$. Gastric mucosal lesions were induced by administering $200 \mathrm{mg} /$ $\mathrm{kg}$ aspirin daily for 4 days. Rats in the mosapride groups were pretreated with mosapride $1 \mathrm{~h}$ prior to aspirin administration. Histological changes were evaluated under a light microscope and gastric epithelial TJs were observed via transmission electron microscopy. The results revealed that cell apoptosis was significantly increased in the aspirin injury group compared with the control $(\mathrm{P}<0.05)$, whereas apoptosis was significantly decreased in the mosapride pretreatment groups compared with the aspirin group $(\mathrm{P}<0.05)$. Cell viability was significantly increased in the mosapride pretreatment groups compared with the aspirin injury group $(\mathrm{P}<0.05)$, and that of the aspirin injury group was significantly decreased compared
\end{abstract}

Correspondence to: Dr Zhenyu Zhang, Department of Gastroenterology, Nanjing First Hospital, Nanjing Medical University, 68 Changle Road, Nanjing, Jiangsu 210006, P.R China E-mail:njzzy808@163.com

*Contributed equally

Key words: mosapride, aspirin, gastric mucosa, tight junction, occludin with the control group $(\mathrm{P}<0.05)$. Compared with the aspirin injury group, occludin expression was significantly increased in the three mosapride pre-treatment groups (all $\mathrm{P}<0.05)$. It was also demonstrated that gastric damage was significantly attenuated in the mosapride pretreatment groups compared with the aspirin injury group $(\mathrm{P}<0.05)$. Impaired TJ integrity was observed in aspirin injury group, whereas TJs in the mosapride groups were almost intact. In conclusion, the results of the present study suggest that mosapride exerts a gastroprotective action on aspirin-induced gastric mucosal injury at least in part via attenuating cell apoptosis and increasing occludin expression.

\section{Introduction}

Non-steroidal anti-inflammatory drugs (NSAIDs), which possess broad analgesic, anti-inflammatory and antipyretic properties, are among the most frequently used drugs in the world (1). Approximately 30 million people worldwide take NSAIDs daily (2) and the use of NSAIDs is increasing due to the widespread existence of pain, inflammation and pyrexia, which require frequent administration of NSAIDs (3). Non-selective NSAIDs, including aspirin, are well known to cause gastrointestinal mucosal damage, particularly in elderly patients or patients who require long-term treatment (4). It has been demonstrated that $2-4 \%$ of non-selective NSAIDs users develop serious gastric mucosal erosions (5) and $20 \%$ of long-term NSAIDs users develop peptic ulcers (6). Selective NSAIDs may be an ideal alternative to traditional NSAIDs as they induce fewer gastrointestinal side effects; however, the use of non-selective NSAIDs remains popular due to their relatively low cost and effectiveness in treating cardiovascular diseases (7). The underlying mechanism by which NSAIDs induce gastric lesions remains unclear. The most probable mechanism is that they disrupt gastric mucosal integrity via the production of free radicals and inhibit production of protective prostaglandins (8). Therefore, studies exploring possible measurements to preserve gastric mucosal integrity in patients on non-selective NSAIDs are required. 
Since the withdrawal of cisapride, the selective 5-hydroxytryptamine-4 receptor (5-HT4R) agonist mosapride has been used as a prokinetic agent for the treatment of dyspeptic symptoms in various gastrointestinal disorders including chronic gastritis, functional dyspepsia (FD), irritable bowel syndrome (IBS) and gastric esophageal reflux disease (GERD) $(9,10)$. Mosapride promotes the release of acetylcholine (Ach) in enteric nerves via activating 5-HT4Rs to stimulate gastrointestinal smooth muscle contraction and motility $(9,10)$. In addition to its prokinetic effect, studies have suggested that mosapride may mediate novel actions on various gastrointestinal injuries by activating 5-HT4R (11-13). A study by Fujisawa et al (11) demonstrated that mosapride attenuated indomethacin-induced gastric mucosal damage in rats. Furthermore, they suggested that this anti-ulcerogenic action of mosapride was mediated via the cholinergic anti-inflammatory pathway. These findings suggest that mosapride has the potential to be used as an anti-ulcerogenic agent for the prevention of ulcers induced by drugs such as NSAIDs or corticosteroids (11). However, the anti-ulcerogenic properties of mosapride have not yet been investigated in larger species and its clinical significance has not been confirmed. The effect of mosapride on gastric mucosal injury remains to be elucidated.

In the present study, the effect of mosapride on aspirin-induced gastric mucosal injury and its effect on the expression of the TJ protein occludin were examined both in vivo and in vitro.

\section{Materials and methods}

Pharmaceuticals. Aspirin was purchased from Sigma-Aldrich (Merck KGaA, Darmstadt, Germany) and mosapride was obtained from Kang Hong Pharmaceuticals Group Co., Ltd. (Chengdu, China).

Cell culture. The human gastric epithelial cell line (GES-1) used in the present study was obtained from the Division of Gastroenterology, Department of Medicine, People's Hospital of Jiangsu Province (Nanjing, China). Cells were cultured in high glucose Dulbecco's modified Eagle medium (DMEM; Hyclone; GE Healthcare Life Sciences, Logan, UT, USA) supplemented with $10 \%$ FBS (Hyclone; GE Healthcare Life Sciences) and $1 \%$ penicillin $\mathrm{G} /$ streptomycin at $37^{\circ} \mathrm{C}$ in a humidified atmosphere containing $5 \% \mathrm{CO}_{2}$. GES-1 cells were seeded at a density of $5 \times 10^{5}$ cells per $75 \mathrm{~cm}^{2}$ culturing flask and were sub-cultured every time they reached $80 \%$ confluence. The culture medium was changed every 48-72 h.

MTT assay. MTT assay was used to examine the effects of mosapride on the viability and proliferation of GES-1 cells Briefly, cells were seeded in 96-well plates at a density of $5 \times 10^{3}$ cells/well in $200 \mu \mathrm{l}$ DMEM and cultured at $37^{\circ} \mathrm{C}$ for $12 \mathrm{~h}$ to achieve attachment. Cells were subsequently divided into 5 groups: Control group (PBS only), aspirin injury group (IC50; $18.32 \mathrm{mmol} / \mathrm{l}$ ) and three mosapride pretreatment groups (18.32 mmol/1 aspirin $+0.4,0.5$ or $0.6 \mu \mathrm{mol} / 1$ mosapride) Cells in the pretreatment groups were treated with mosapride for $6 \mathrm{~h}$ and subsequently cultured at $37^{\circ} \mathrm{C}$ with aspirin for $12 \mathrm{~h}$. Following this, MTT solution $(5 \mathrm{mg} / \mathrm{ml}$; Sigma-Aldrich; Merck $\mathrm{KGaA}$ ) was added to each well and incubated at $37^{\circ} \mathrm{C}$ for $4 \mathrm{~h}$. The culture medium was removed, dimethylsulfoxide (DMSO) was added to each well (200 $\mu \mathrm{l} /$ well; Sigma-Aldrich; Merck KGaA) and cells were incubated at $37^{\circ} \mathrm{C}$ for $10 \mathrm{~min}$, following which the absorbance (A) of MTT was measured at $570 \mathrm{~nm}$ using a microplate reader (Bio-Rad Laboratories, Inc., Hercules, CA, USA). The control group was used to set zero point. The inhibition rate (IR) was calculated using the following equation: $\mathrm{IR}=\left(\mathrm{A}_{\text {control }}-\mathrm{A}_{\text {experiment }}\right) / \mathrm{A}_{\text {control }} \times 100$. The IC50 values were calculated using a probit model and all experiments were repeated $\geq 3$ times.

Flow cytometry analysis. Apoptosis was determined by fluorescein isothiocyanate (FITC)-labeled Annexin V/propidium iodide (PI) double staining and flow cytometry analysis. A FITC-labeled Annexin V/PI apoptosis assay kit (Nanjing KeyGen Biotech Co., Ltd., Nanjing, China) was used, according to the manufacturer's instructions. Cells in the control group were treated with vehicle $(0.1 \%$ DMSO in the working medium), and the four treatment groups were treated with aspirin with or without different doses of mosapride as described above. Cells were centrifuged at 1,500 x $g$ for $5 \mathrm{~min}$ $\left(4^{\circ} \mathrm{C}\right)$ and subsequently washed twice with PBS. Following this, cells were resuspended $\left(1 \times 10^{6}\right.$ cells $\left./ \mathrm{ml}\right)$ in binding buffer (a hypotonic solution containing $0.1 \%$ sodium citrate, $0.1 \%$ Triton X-100 and $50 \mu \mathrm{g} / \mathrm{ml}$ PI; Nanjing KeyGen Biotech Co., Ltd.) and incubated for $30 \mathrm{~min}$ at $4^{\circ} \mathrm{C}$. Aliquots containing $1 \times 10^{5}$ cells in $100 \mu \mathrm{l}$ buffer were stained with $10 \mu \mathrm{l} \mathrm{PI}(50 \mu \mathrm{g} / \mathrm{ml})$ solution and $5 \mu \mathrm{l}$ fluorescein isothiocyanate-conjugated AV $(17.6 \mu \mathrm{g} / \mathrm{ml})$ at $37^{\circ} \mathrm{C}$ for $5 \mathrm{~min}$ in darkness. Following staining, $400 \mu \mathrm{l}$ of binding buffer was added to the cells before detection via flow cytometry assay (FACSCalibur; BD Biosciences, San Jose, CA, USA). The data were analyzed using CellQuest v.5.1 software (BD Biosciences). Detections were completed within $1 \mathrm{~h}$. The experiments were performed in triplicate.

Western blot analysis. Total proteins were extracted from GES-1 cells using a protein extraction kit (KGP350-2/ KGP3100-2; Nanjing KeyGen Biotech Co., Ltd.), according to the manufacturer's instructions. Protein concentrations were determined using a bicinchoninic acid assay kit (Nanjing KeyGen Biotech Co., Ltd.). Samples mixed with loading buffer (radioimmunoprecipitation assay buffer; Nanjing KeyGen Biotech Co., Ltd.) were boiled at $100^{\circ} \mathrm{C}$ for $5 \mathrm{~min}$ to denature and protein concentration was subsequently determined. Briefly, equal amounts of proteins (50 $\mu \mathrm{g} / \mathrm{lane})$ were separated by $12 \%$ SDS-PAGE following instantaneous centrifugation at $12,000 \times \mathrm{g}$ for $15 \mathrm{~min}\left(4^{\circ} \mathrm{C}\right)$. Blots were subsequently transferred onto polyvinylidene membranes and nonspecific protein binding was blocked by incubation with $5 \%$ skimmed milk in TBST $1 \mathrm{X}$ containing $0.5 \%$ Tween-20 for $2 \mathrm{~h}$ at room temperature. Membranes were subsequently incubated at $4^{\circ} \mathrm{C}$ overnight with primary anti-occludin $(1: 1,000$; sc-271842; Santa Cruz Biotechnology, Inc., Dallas, TX, USA) and anti- $\beta$-actin $(1: 1,000 ; 4970$; Cell Signaling Technology, Inc., Danvers, MA, USA) antibodies. Membranes were then thoroughly washed with TBST buffer and incubated with the corresponding horseradish peroxidase-conjugated secondary occludin (1:200; sc-516141; Santa Cruz Biotechnology, Inc.) and $\beta$-actin $(1: 1,000 ; 7075$; Cell Signaling Technology, Inc.) antibodies for $2 \mathrm{~h}$ at $24^{\circ} \mathrm{C}$. Membranes were washed with TBST 
a further 3 times and the blot was detected using an enhanced chemiluminescence system (Nanjing KeyGen Biotech Co., Ltd.). Protein band density was semi-quantified using the Gel and Graph Digitizing system (Silk Scientific, Inc., Orem, UT, USA). All experiments were performed in triplicate.

Animals. A total of 40 2-3-month-old male Sprague-Dawley rats (weight, 200-250 g) were provided by the Laboratory Animal Center of Nanjing First Hospital Affiliated to Nanjing Medical University (Nanjing, China). Rats were housed in cages under pathogen-free conditions maintained at a constant temperature of $23^{\circ} \mathrm{C}$, a constant relative humidity of $50-70 \%$ and a 12-h light/dark cycle. Rats were acclimatized for at least one week prior to commencement of the experiment. All rats had ad libitum access to standard rodent chow and water. Prior to the treatment, the rats were fasted for $24 \mathrm{~h}$, but had free access to water. All procedures involved in the animal experiments were approved by the Committee on the Ethics of Animal Experiments of Nanjing Medical University.

Induction of gastric mucosal lesions by aspirin. Rats were randomized into five groups ( $n=8$ per group): Control group, aspirin injury group and mosapride pretreatment groups $(0.25,0.50$ or $0.75 \mathrm{mg} / \mathrm{kg})$. Gastric lesions were induced by the administration of aspirin $(200 \mathrm{mg} / \mathrm{kg}$ ) and mosapride was administered $1 \mathrm{~h}$ prior to aspirin administration during a consecutive period of 4 days. Aspirin and mosapride were suspended in $0.5 \%$ carboxymethylcellulose (CMC; Tianjin Kemiou Chemical Reagent Co., Ltd. Tianjin, China). Rats in the control group were given $5 \mathrm{ml} / \mathrm{kg} 0.5 \% \mathrm{CMC}$ solution. On the fourth day of the experiment, all rats were humanely sacrificed by anesthetization with pentobarbital $(100 \mathrm{mg} / \mathrm{kg}$ body weight; Nanjing KeyGen Biotech Co., Ltd.) 8 h following the administration of pharmacological agents or vehicle. The stomach was then removed and parameters were scored.

Assessment of gastric lesions. The gastric mucosa was exposed by cutting the stomach along the greater curvature, washing it with saline and laying it on a flat wooden board. The macroscopic assessment of aspirin-induced gastric lesions was performed by two independent examiners blinded to the treatment the rats had received. The assessment of lesions was made according to a semi-quantitative scale as previously described by Guth (14).

Histological analysis. Gastric tissue specimens were fixed in $10 \%$ buffered formalin for $24 \mathrm{~h}$ at room temperature and processed in a paraffin tissue-processing machine (CUT 4062; SLEE Medical GmbH, Mainz, Germany). The stomach was sliced into sections $5 \mu \mathrm{m}$ thick and stained with hematoxylin and eosin for histological evaluation. Changes in gastric morphology were subsequently observed under a microscope (CX41; Olympus Corporation, Tokyo, Japan).

Transmission electron microscopy. Transmission electron microscopy was used to detect alterations in tight junction (TJ) ultrastructure. Specimens from the gastric tissues were washed three times in $0.1 \mathrm{~mol} / \mathrm{l}$ cacodylate buffer and then fixed with $2.5 \%$ glutaraldehyde and $1 \%$ osmic acid (Nanjing KeyGen Biotech Co., Ltd.) at room temperature for $1 \mathrm{~h}$, and then at $4^{\circ} \mathrm{C}$ for 4 days. Subsequently, tissues were washed with $0.1 \mathrm{~mol} / 1$ cacodylate buffer at room temperature for $10 \mathrm{~min}$, treated with $2 \%$ osmium tetroxide in $0.1 \mathrm{~mol} / 1 \mathrm{caco}-$ dylate buffer for $1 \mathrm{~h}$ at room temperature and washed with $0.1 \mathrm{~mol} / 1$ cacodylate buffer for $10 \mathrm{~min}$ at room temperature. Following this, tissues were washed with distilled water for $10 \mathrm{~min}$ at room temperature and then dehydrated through a graded ethanol series (30-100\%). The tissues were incubated for $5 \mathrm{~min}$ at room temperature in $100 \%$ ethanol and then embedded in epoxy resin Epon 618 (Nanjing KeyGen Biotech Co., Ltd.). Ultrathin sections (90 nm) were obtained and mounted on copper grids and double stained with saturated uranyl acetate in $50 \%$ ethanol, followed by $0.4 \%$ lead citrate at $37^{\circ} \mathrm{C}$ for $12 \mathrm{~h}$. The changes in TJs were observed with a transmission electron microscope (JEM-200; JEOL, Ltd., Tokyo, Japan).

Statistical analysis. All data are expressed as the mean \pm standard deviation. One-way analysis of variance and Student's $t$-tests were performed. All statistical analysis was performed using SPSS 19.0 (IBM SPSS, Armonk, NY, USA) and $\mathrm{P}<0.05$ was considered to indicate a statistically significant difference.

\section{Results}

Mosapride protect GES-1 cells against aspirin-induced cytotoxicity and apoptosis. MTT and AV/PI flow cytometry analyses were performed to determine the effect of mosapride on apoptosis of GES-1 cells. As shown in Fig. 1, aspirin significantly induced the apoptosis of GES-1 cells $(\mathrm{P}<0.05)$ compared with the control group and mosapride significantly attenuated the aspirin-induced apoptosis, most notably at a dose of $0.5 \mu \mathrm{mol} / 1$, compared with the aspirin injury group $(\mathrm{P}<0.05)$.

Mosapride upregulates the expression of TJ protein occludin against aspirin-induced injury in GES-1 cells. Protein levels of TJ occludin were significantly decreased compared with the control group following $12 \mathrm{~h}$ treatment with aspirin as determined by western blotting $(\mathrm{P}<0.05$; Fig. 2$)$. However, this effect was significantly attenuated by pretreatment with mosapride at all doses, most notably at a dose of $0.5 \mu \mathrm{mol} / 1$ ( $\mathrm{P}<0.05$; Fig. 2).

Histological evaluation of gastric lesions: Administration of mosapride significantly reduces the evidence of gastric ulcers and тисоsal erosions. Rats were pretreated with various doses of mosapride $(0.25,0.50$ or $0.75 \mathrm{mg} / \mathrm{kg}) 1 \mathrm{~h}$ prior to aspirin administration. At $8 \mathrm{~h}$ after administration, rats were sacrificed and gastric mucosal lesions were assessed. Pretreatment with mosapride was found to significantly reduce gastric mucosal lesion scores compared with the control aspirin group (Fig. 3A; $\mathrm{P}<0.05$ ).

Histological changes were observed with a light microscope. Histological observation revealed that aspirin treatment markedly induced gastric ulcers and mucosa erosions, and mucosa lost their normal structure and integrity, the glandular structure was destroyed and visible inflammatory cell infiltration was observed (Fig. 3Bb). These histological changes were 
A

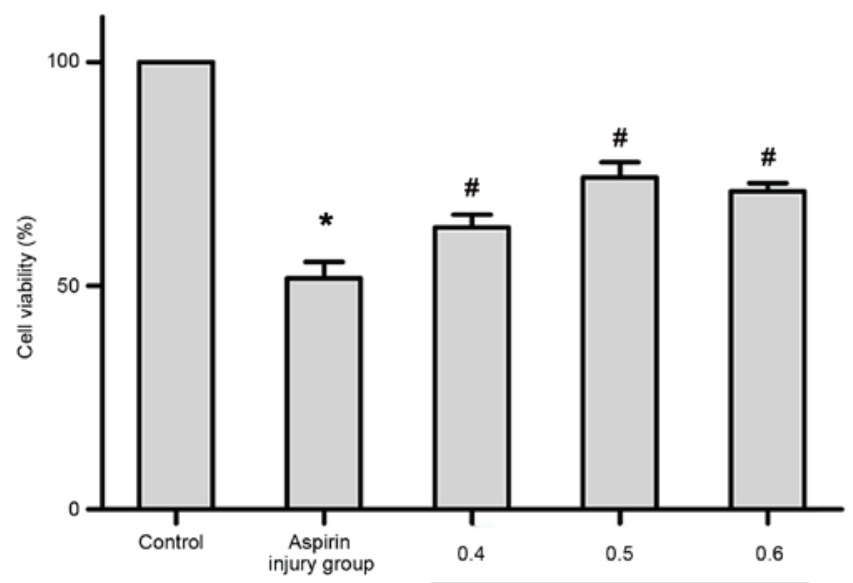

B

Mosapride $(\mu \mathrm{mol} /)$
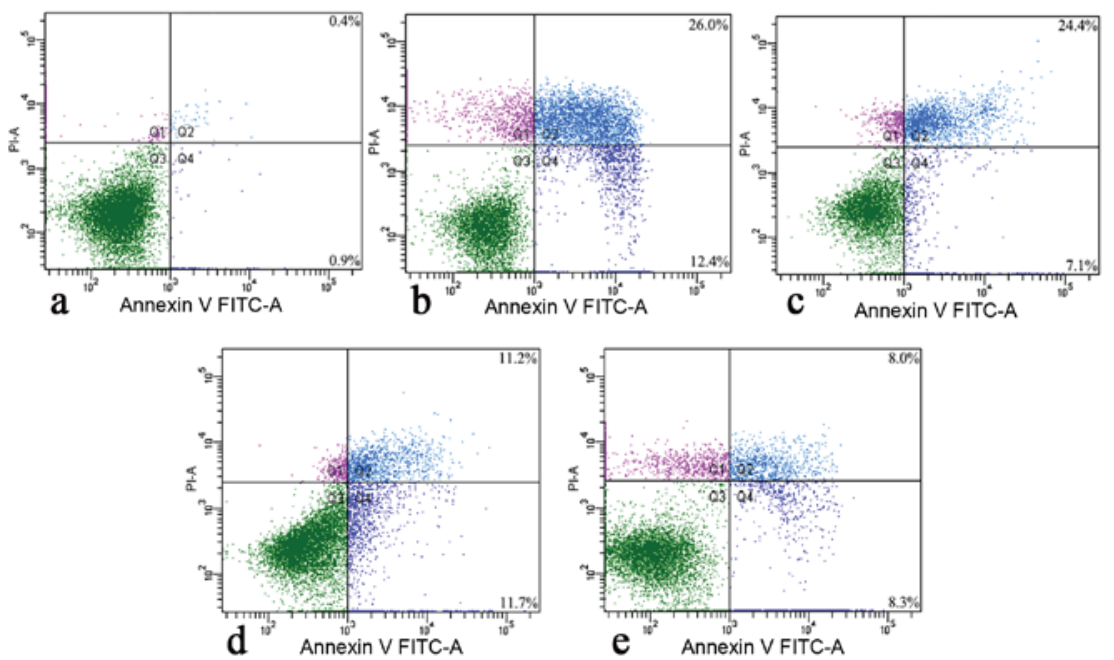

Figure 1. Effects of mosapride on GES-1 cells against aspirin-induced cytotoxicity. Pretreatment with mosapride $(0.4,0.5$, or $0.6 \mu \mathrm{mol} / \mathrm{l})$ promotes cell (A) viability and (B) proliferation in an aspirin-induced cytotoxicity model. Data are presented as the mean \pm standard deviation. ${ }^{*} \mathrm{P}<0.05$ vs. control group; ${ }^{\sharp} \mathrm{P}<0.05$ vs. aspirin injury group. FITC, fluorescein isothiocyanate; PI, propidium iodide.
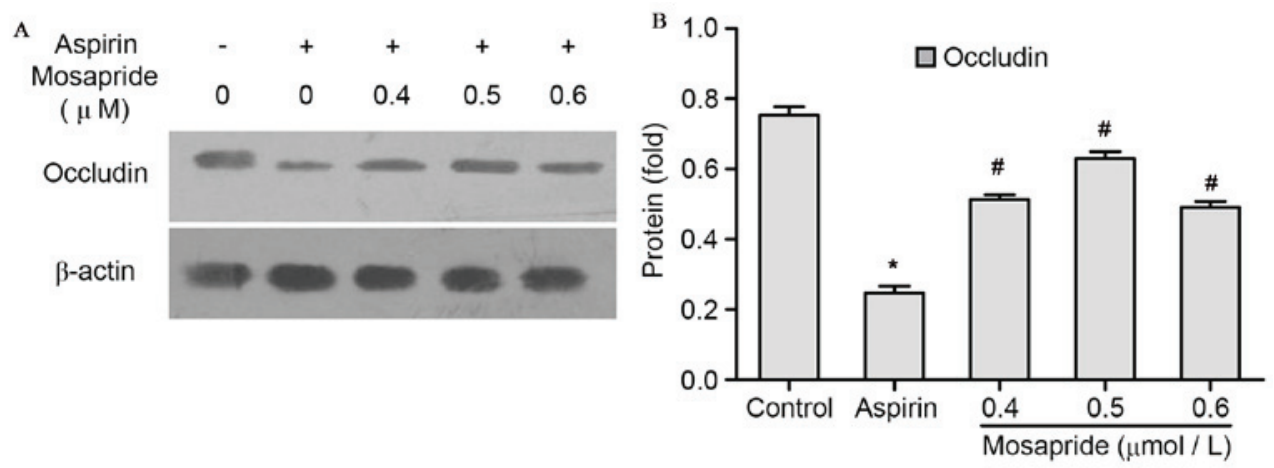

Figure 2. Effects of pretreatment with mosapride on occludin protein expression in GES-1 cells. (A) Western blot analysis and (B) quantification of western blot analysis results. Administration of aspirin ( $18.32 \mathrm{mM}$ for $12 \mathrm{~h}$ ) downregulated occludin expression. This effect was attenuated by mosapride pretreatment $(0.4,0.5,0.6 \mu \mathrm{M}$ for $6 \mathrm{~h})$ with the $0.5 \mu \mathrm{M}$ being the most potent dose. Data are presented as the mean + standard deviation. ${ }^{*} \mathrm{P}<0.05 \mathrm{vs}$. control group; ${ }^{*} \mathrm{P}<0.05$ vs. aspirin group.

partly attenuated by treatment with mosapride; the degree of injuries was reduced and inflammatory cell infiltration decreased in the gastric mucosa (Fig. 3Bc-e), most notably at a dose of $0.50 \mathrm{mg} / \mathrm{kg}$ (Fig. 3Bd). A protective effect was seen for all doses of mosapride, with the medium-dose being the most potent in terms of reducing the number, size and score of mucosal lesions.

Effects of mosapride on aspirin-induced ultrastructure alterations of TJ in rats. Transmission electron microscopy 


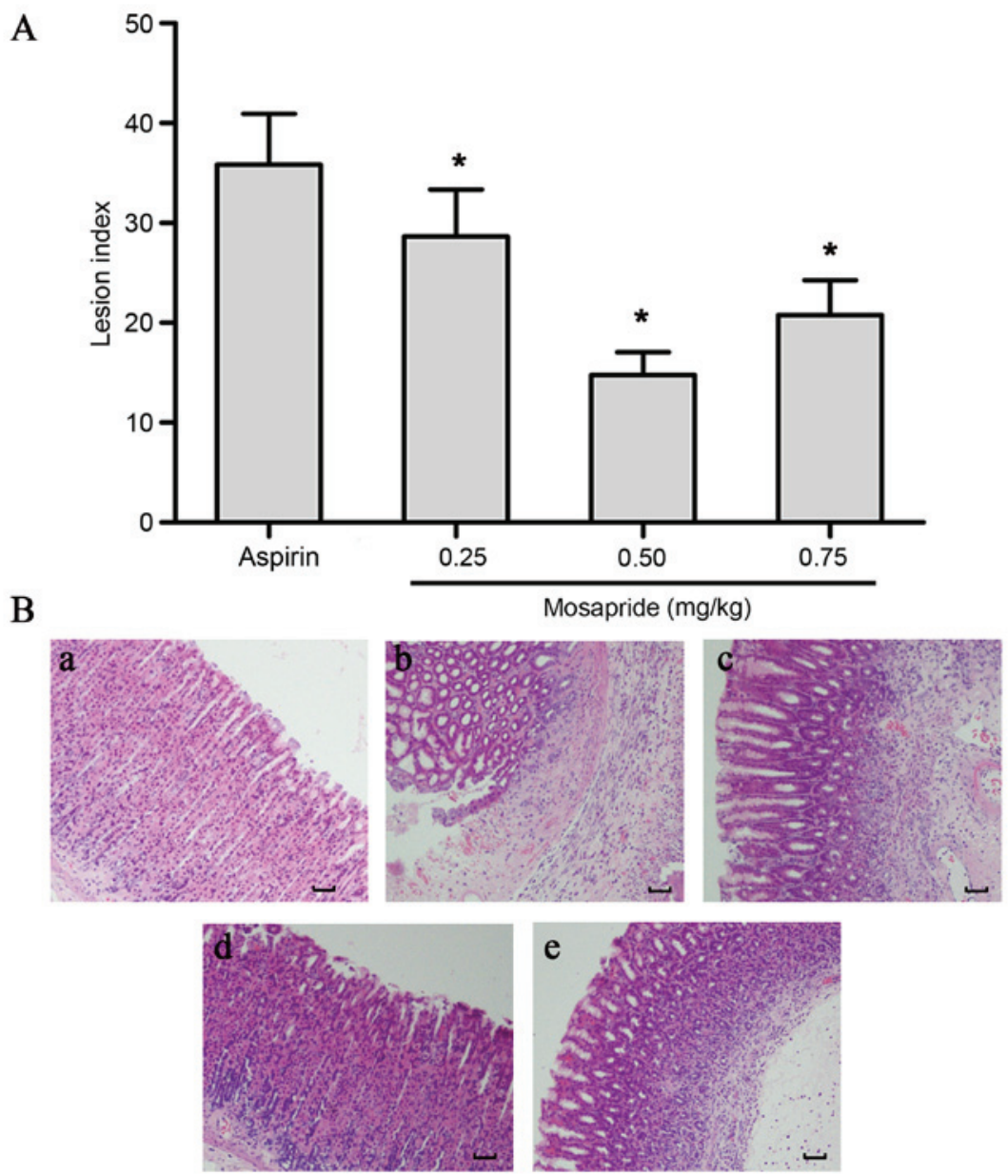

Figure 3. Effects of mosapride pretreatment on aspirin-induced gastric lesions in rats and histological observation. (A) The lesion index was measured and recorded by Guth's standard. (B) In the control group, (Ba) gastric mucosal integrity was observed. In the aspirin injury group, (Bb) the gastric mucosa was severely damaged, characterized by broken glandular structural and obvious granulocyte infiltration. Pretreatment with (Bc) 0.25 , (Bd) $0.5 \mathrm{or}(\mathrm{Be}) 0.75 \mathrm{mg} / \mathrm{kg}$ mosapride markedly attenuated aspirin-induced gastric lesions, most notably at a dosage of $0.5 \mathrm{mg} / \mathrm{kg}$. Data are presented as the mean \pm standard deviation. "P<0.05 vs. aspirin group. Hematoxylin and eosin staining. Magnification, x100. Scale bar, $1 \mathrm{~mm}$.
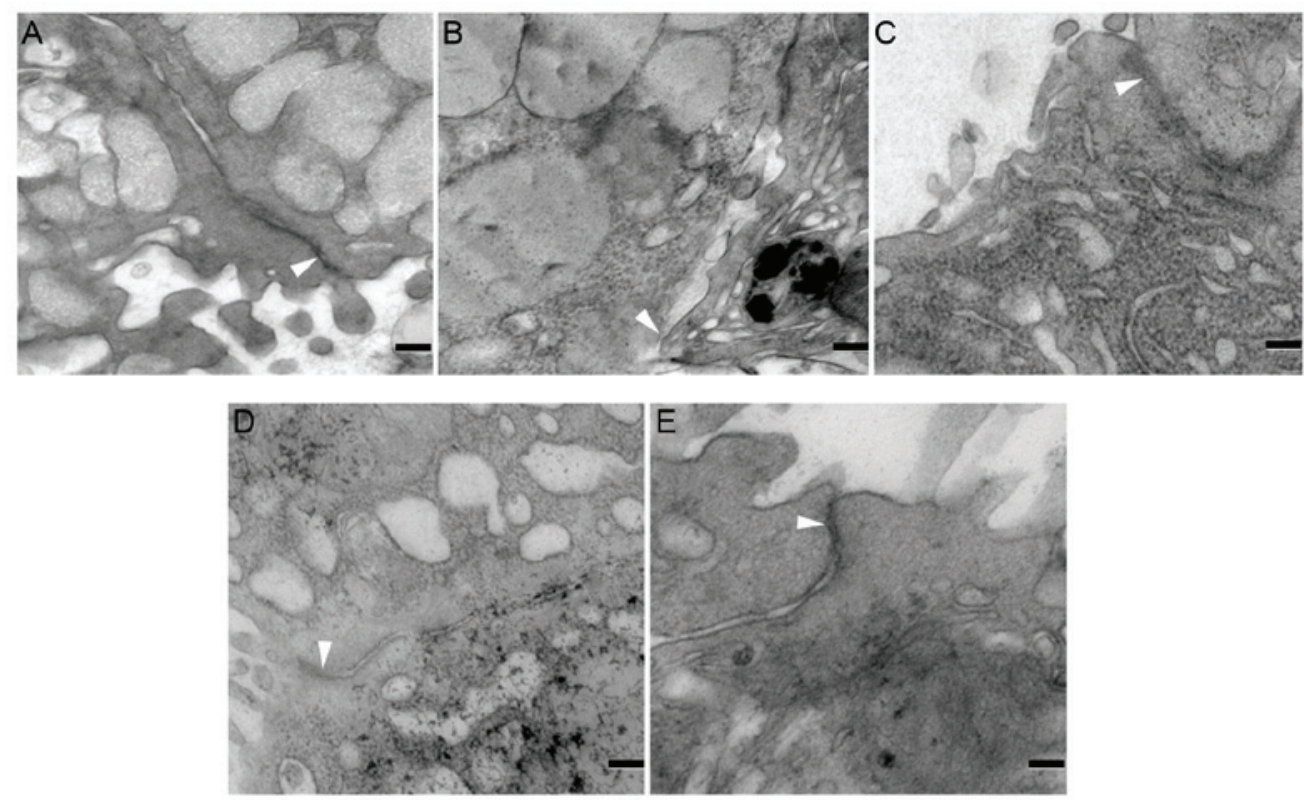

Figure 4. Effects of mosapride on TJ protection ultrastructure detected by transmission electron microscopy. (A) TJ structures were intact in the control group. (B) Aspirin severely disrupted the TJ ultrastructure, characterized by decreased electron-dense materials in the TJ and loss of TJ membrane fusion, further widened gaps and tracer extravasation. Pretreatment with (C) 0.4 , (D) 0.5 or (E) $0.6 \mathrm{mg} / \mathrm{kg}$ mosapride markedly alleviated the effects of aspirin. White arrowheads indicate typical changes in TJ structure, including widened gaps and tracer extravasation. Scale bar, $0.5 \mu \mathrm{m}$. TJ, tight junction. 
analysis was performed to evaluate the influence of aspirin and mosapride on TJ ultrastructure. In the control group, the structure of TJs was intact (Fig. 4A), whereas TJ ultrastructure was markedly disrupted by aspirin administration, characterized by decreased electron-dense material in the TJ and a loss of TJ membrane fusion (Fig. 4B). Furthermore, widened gaps and tracer extravasation were observed (Fig. 4B), indicating a decrease in TJ integrity. This effect was markedly attenuated by mosapride pretreatment (Fig. 4C-E).

\section{Discussion}

Previous studies have been performed to investigate pharmacological agents that have various antiulcer healing properties for aspirin-induced gastric lesions $(11,15)$. In the present study, the effects of mosapride on healing action of the gastric mucosal and the expression of the TJ protein occludin were investigated both in vivo and in vitro. To the best of our knowledge, there have been no previous studies investigating the healing effect of mosapride on aspirin-induced gastric lesions.

NSAIDs are among the most widely used medicines worldwide due to their anti-inflammatory, antipyretic and analgesic properties (1). However, studies have revealed that NSAIDs can cause various side effects in the gastrointestinal tract, including gastrointestinal ulcers, bleeding and perforation, the complications of which may be fatal $(2,3,6)$. A previous study suggested that injury caused by aspirin is different to injuries caused by indomethacin and other NSAIDs (16). The exogenous prostaglandin (PG) analogue misoprostol has a significant curative effect on gastric mucosa damage induced by indomethacin; however, when it comes to lesions caused by aspirin, the effect is minimal, even with large doses of misoprostol $(15,16)$. It has been suggested that impaired TJ integrity may increase gastric permeability, thus contributing to aspirin-induced gastric mucosal injury (16). In the present study, it was demonstrated that aspirin is able to cause a significant downregulation in occludin in GES-1 cells, as determined by western blot analysis, and obvious damage to TJ integrity as detected via transmission electron microscopy in a rat model.

Scientists have discovered that 5-HT4 receptor agonists have an unexpected gastroprotective effect (12). A study by Alarcon et al (17) demonstrated that the nonselective 5-HT4 receptor agonist cisapride is able to resist gastric mucosal injury induced by ethanol and stress. In this study, gastric ulceration was significantly relieved and the hexosamine content of gastric mucus was significantly increased, as were the gastric mucosal levels of PGE2 $(12,17)$. However, cisapride is associated with adverse cardiovascular events and has therefore been replaced by mosapride, a novel selective 5 -HT4 receptor agonist with safer profiles $(18,19)$. A study by Fujisawa et al (11) found that treatment with a low dose of mosapride inhibited gastric mucosa injury induced by indomethacin, and their results showed that $0.5 \mathrm{mg} / \mathrm{kg}$ mosapride was the optimal dose. At this dosage, the gastric emptying mobility is not activated whereas the release of ACh from parasympathetic nerves is accelerated via the activation of 5-HT4 receptors, which is followed by a cascade and activation of the nicotinic anti-inflammatory system (11). When methyllycaconitine, a selective inhibitor of Ach receptors, was added, the anti-ulcerogenic activity of mosapride was abrogated. Kato et al (20) suggested that endogenous 5-HT has both pro- and anti-ulcerogenic effects on the pathogenesis of indomethacin-induced small intestinal damage via activation of 5-HT3 and 5-HT4 receptors, respectively, and that the 5-HT4 receptor agonist mosapride can protect against the occurrence of these lesions in a dose-dependent manner. In the present study, aspirin inhibited the proliferation of the GES-1 cell line with an IC50 value of $18.32 \mathrm{mmol} / \mathrm{l}$. When cells were pretreated with mosapride, the apoptotic cell ratio was significantly lower in the experiment group compared with the control group, as indicated by flow cytometry. The maximum protective effect was achieved at a dose of $0.5 \mu \mathrm{mol} / 1$. The in vivo part of the present study also demonstrated that mosapride was able to attenuate aspirin-induced gastric injury.

The protective gastric epithelial barrier comprises three components: The compact epithelial cell lining, the specialized mucus covering and the bicarbonate ions (21). Alongside the gastric epithelial cell layer there exists a junctional complex, typically composed of TJs, intermediate junctions, desmosomes and gap junctions (21). Of these, TJs are the most critical in binding the epithelial cell layers and thus separating physiologically distinct compartments, restricting luminal antigens (21). TJs separate the apical cell surface domains from the basolateral cell surface domains, so that cell polarity can be established and maintained, and a paracellular permeability barrier can be established to inhibit solute and water flow through the paracellular space (22). It has previously been demonstrated that multiple proteins, including occludin, claudins, zona occludens and junctional adhesion molecules are involved in the formation of TJs $(23,24)$. At present, occludin and claudins are considered to be major functional proteins. Occludin is a transmembrane protein with a molecular weight of $65 \mathrm{KDa}(25)$, and its level of expression is correlated with the number of TJ strands in a variety of epithelia $(26,27)$, such as the gastrointestinal tract epithelium. In the present study, western blot analysis was used to determine the expression of occludin, and it was demonstrated that treating GES-1 cells with aspirin decreased the expression of occludin. This aspirin-induced reduction of occludin was reversed by pretreatment with mosapride. To further examine the impaired TJs, transmission electron microscopy was utilized to observe TJ alterations in a gastric mucosal in a rat model. The results showed that TJ integrity was markedly reduced by the administration of aspirin and that pretreatment of mosapride was able to partially protect TJs from drastic damage caused by aspirin. This is in agreement with a previous study carried out by Fujisawa et al (11). The present study also visualized alterations in the TJ ultrastructure and confirmed this effect at a cellular level. However, further research is required to fully elucidate the underlying mechanisms of this.

There were some major limitations to the present study that are worth careful consideration. Firstly, only the GES-1 cell line was investigated; to be sure about the protective role against aspirin-induced damage at the cellular level, other cell lines need to be investigated. Secondly, occludin was selected as a representative molecule to assess the integrity of TJs. Other molecules should be considered and investigated in future studies. Thirdly, the gastroprotective effect of mosapride on aspirin-induced injury was only observed in terms of cell 
apoptosis and viability in vivo, and morphological changes in vitro. The signaling pathway triggered by mosapride was not thoroughly investigated in the present study, and further studies are crucial to fully understand the underlying mechanisms.

In conclusion, the results of the present study demonstrate that aspirin causes damage in GES-1 cells and induces gastric lesions in a rat model, collectively leading to a decrease in occludin expression and impairing TJ integrity. These effects may be partially reversed by pretreatment with mosapride, which may function by upregulating occludin expression to help restore the gastric mucosal epithelial barriers. Further studies are necessary to confirm these findings in relevant clinical cases and the exact underlying mechanism of this protective effect remains to be elucidated.

\section{Acknowledgements}

The authors of the present study would like to thank Qiong-Yu Mi, Shu-Li Zhao and Yu-Qin Pan (Nanjing First Hospital Central Laboratory, Nanjing, China) for their excellent technical assistance.

\section{References}

1. Harirforoosh S, Asqhar W and Jamali F: Adverse effects of nonsteroidal antiinflammatory drugs: An update of gastrointestinal, cardiovascular and renal complications. J Pharm Pharm Sci 16: 821-847, 2013

2. Maiden L: Capsule endoscopic diagnosis of nonsteroidal antiinflammatory drug-induced enteropathy. J Gastroenterol 44 (Suppl 19): 64-71, 2009.

3. Hegazy R, Alashhab M and Amin M: Cardiorenal effects of newer NSAIDs (Celecoxib) versus classic NSAIDs (Ibuprofen) in patients with arthritis. J Toxicol 2011: 862153, 2011

4. Allison MC, Howatson AG, Torrance CJ, Lee FD and Russell RI Gastrointestinal damage associated with the use of nonsteroidal antiinflammatory drugs. N Engl J Med 327: 749-754, 1992.

5. Goldstein JL, Larson LR, Yamashita BD and Boyd MS: Management of NSAID-induced gastropathy: An economic decision analysis. J Clin Ther 19: 1496-1509, 1424-1425, 1997.

6. Naesdal J and Brown K: NSAID-associated adverse effects and acid control aids to prevent them: A review of current treatment options. J Drug Saf 29: 119-132, 2006.

7. Mukherjee D, Nissen SE and Topol EJ: Risk of cardiovascular events associated with selective COX-2 inhibitors. JAMA 286: 954-959, 2001

8. Hawkey CJ: The gastroenterologist's caseload: Contribution of the rheumatologist. Semin Arthritis Rheum 26 (6 Suppl 1): S11-S15, 1997.

9. Curran MP and Robinson DM: Mosapride in gastrointestinal disorders. Drugs 68: 981-991, 2008.

10. Odaka T, Suzuki T, Seza A, Yamaguchi T and Saisho H: Serotonin 5-HT4 receptor agonist (mosapride citrate). Nihon Rinsho 64: 1491-1494, 2006 (In Japanese).

11. Fujisawa M, Murata T, Hori M and Ozaki H: The 5-HT4 receptor agonist mosapride attenuates NSAID-induced gastric mucosal damage. J Gastroenterol 45: 179-186, 2010.
12. Alarcón-de-la-Lastra Romero C, López A, Martín MJ, la Casa C and Motilva V: Cinitapride protects against ethanol-induced gastric mucosal injury in rats: Role of 5-hydroxytryptamine, prostaglandins and sulfhydryl compounds. J Pharmacology 54: 193-202, 1997

13. Bonaz B: The cholinergic anti-inflammatory pathway and the gastrointestinal tract. Gastroenterology 133: 1370-1373, 2007.

14. Guth PH, Aures D and Paulsen G: Topical aspirin plus $\mathrm{HCl}$ gastric lesions in the rat. Cytoprotective effect of prostaglandin, cimetidine, and probanthine. Gastroenterology 76: 88-93, 1979.

15. Meyer RA, McGinley D and Posalaky Z: The effects of 16 , 16-dimethyl prostaglandin E2 + aspirin on the canine gastric mucosal barrier. Virchows Arch A Pathol Anat Histopathol 412: $119-125,1987$.

16. Oshima T, Miwa H and Joh T: Aspirin induces gastric epithelial barrier dysfunction by activating p38 MAPK via claudin-7. Am J Physiol Cell Physiol 295: C800-C806, 2008.

17. Alarcón de la Lastra C, Martin MJ, La Casa M, López A and Motilva V: Effects of cisapride on ulcer formation and gastric secretion in rats: Comparison with ranitidine and omeprazol. Gen Pharmacol 27: 1415-1420, 1996.

18. Quigley EM: Cisapride: What can we learn from the rise and fall of a prokinetic? J Dig Dis 12: 147-156, 2011.

19. Tack J, Camilleri M, Chang L, Chey WD, Galligan JJ, Lacy BE, Müller-Lissner S, Quigley EM, Schuurkes J, De Maeyer JH and Stanghellini V: Systematic review: Cardiovascular safety profile of 5-HT(4) agonists developed for gastrointestinal disorders. Aliment Pharmacol Ther 35: 745-767, 2012.

20. Kato S, Matsuda N, Matsumoto K, Wada M, Onimaru N, Yasuda M, Amagase K, Horie S and Takeuchi K: Dual role of serotonin in the pathogenesis of indomethacin-induced small intestinal ulceration: Pro-ulcerogenic action via 5-HT3 receptors and anti-ulcerogenic action via 5-HT4 receptors. Pharmacol Res 66: 226-234, 2012.

21. Berkes J, Viswanathan VK, Savkovic SD and Hecht G: Intestinal epithelial responses to enteric pathogens: Effects on the tight junction barrier, ion transport, and inflammation. Gut 52: 439-451, 2003

22. Tsukita S and Furuse M: Pores in the wall: Claudins constitute tight junction strands containing aqueous pores. J Cell Biol 149: 13-16, 2000.

23. Furuse M, Fujita K, Hiiragi T, Fujimoto K and Tsukita S: Claudin-1 and -2: Novel integral membrane proteins localizing at tight junctions with no sequence similarity to occludin. J Cell Biol 141: 1539-1550, 1998.

24. Martin-Padura I, Lostaglio S, Schneemann M, Williams L, Romano M, Fruscella P, Panzeri C, Stoppacciaro A, Ruco L, Villa A, et al: Junctional adhesion molecule, a novel member of the immunoglobulin superfamily that distributes at intercellular junctions and modulates monocyte transmigration. J Cell Biol 142: 117-127, 1998.

25. Tsukita S and Furuse M: Occludin and claudins in tight-junction strands: Leading or supporting players? Trends Cell Biol 9: 268-273, 1999

26. Schulzke JD and Fromm M: Tight junctions: Molecular structure meets function. Ann N Y Acad Sci 1165: 1-6, 2009.

27. Medina R, Rahner C, Mitic LL, Anderson JM and Van Itallie CM: Occludin localization at the tight junction requires the second extracellular loop. J Membr Biol 178: 235-247, 2000. 